\title{
Genetic algorithm-assisted soft fusion-based linear cooperative spectrum sensing
}

\author{
Ayman A. El-Saleh ${ }^{1,2 a)}$, Mahamod Ismail ${ }^{1}$, \\ and Mohd Alaudin Mohd Ali ${ }^{1}$ \\ ${ }^{1}$ Department of Electrical, Electronics, and Systems Engineering, University \\ Kebangsaan Malaysia, 43600 Bangi, Selangor, Malaysia \\ ${ }^{2}$ Faculty of Engineering, Multimedia University, Jalan Multimedia, 63100 \\ Cyberjaya, Selangor, Malaysia \\ a)ayman.elsaleh@mmu.edu.my
}

\begin{abstract}
In this letter, a cooperative spectrum sensing scheme for cognitive radio networks based on genetic algorithm (GA) is proposed. The proposed scheme lies in optimizing the weighting coefficients vector used in a linear soft-decision fusion- (SDF-) based framework. The detection performance of the proposed GA-assisted SDF-based scheme is compared with the conventional SDF schemes as well as the OR-logic hard-decision fusion (HDF) scheme. The simulation results show that the proposed scheme outperforms all other schemes and can achieve higher probability of detection given the same probability of false alarm.
\end{abstract}

Keywords: cognitive radio, spectrum sensing, genetic algorithm, SDF, HDF

Classification: Wireless circuits and devices

\section{References}

[1] S. Haykin, "Cognitive radio: Brain-empowered wireless communications," IEEE J. Sel. Areas Commun., vol. 23, no. 2, pp. 201-220, Feb. 2005.

[2] A. Ghasemi and E. S. Sousa, "Collaborative spectrum sensing for opportunistic access in fading environments," Proc. IEEE DySPAN, Baltimore, MD, USA, pp. 131-136, 2005.

[3] W. Zhang, R. K. Mallik, and K. B. Letaief, "Cooperative spectrum sensing optimization in cognitive radio networks," Proc. IEEE Int. Conf. Commun, pp. 3411-3415, 2008.

[4] Z. Quan, S. Cui, and A. H. Sayed, "Optimal linear cooperation for spectrum sensing in cognitive radio networks," IEEE J. Sel. Topics Signal Processing, vol. 2, no. 1, 2008.

[5] B. Shen and K. S. Kwak, "Soft combination schemes for cooperative spectrum sensing in cognitive radio networks," ETRI Journal, vol. 31, no. 3, 2009.

[6] A. A. El-Saleh, M. Ismail, M. A. M. Ali, and I. H. Arka, "Hybrid SDFHDF cluster-based fusion scheme for cooperative spectrum sensing in cognitive radio networks," KSII Trans. Internet and Info. Sys., vol. 4, no. 6, pp. 1023-1041, Dec. 2010. 
[7] M. Gen and R. Cheng, Genetic Algorithms and Engineering Optimization, John Wiley \& Sons, 2000.

[8] J. J. Grefenstette, "Optimization of control parameters for genetic algorithms," IEEE Trans. Sys., Man, Cybern., vol. SMC-16, no. 1, pp. 122$128,1986$.

\section{Introduction}

In today's wireless communications, protection from interference is guaranteed by licensing radio spectrum for exclusive usage. As a result of such static allocation policy, large segments of the spectrum are currently used inefficiently. Even more problematic, the demand for additional spectrum is growing faster than the technology is able to increase spectrum efficiency. These shortages emerged the idea of dynamic spectrum access (DSA), which is a technology that senses open channels and allows devices to communicate in underused parts of the spectrum [1]. Cognitive radios (CRs) realize the DSA concept as they autonomously and opportunistically coordinate the usage of spectrum. CRs identify radio spectrum when it is unused by the incumbent radio, or primary user (PU) system, and use this spectrum in an intelligent way based on spectrum observation [1]. Incumbent awareness and interference prevention are partially realized as part of MAC and PHY layers by means of cooperative spectrum sensing, which is used to alleviate the so-called hidden terminal problem in shadowing environments [2]. Cooperative spectrum sensing can be deployed as hard-decision fusion (HDF) schemes [3] or soft-decision fusion (SDF) schemes [4, 5]. In [4, 5], linear SDFbased cooperative spectrum sensing was proposed and the optimal weighting coefficients vector of all $\mathrm{CR}$ systems in the network was derived by maximizing the deflection coefficient (DC). The DC provides a good measure of the detection performance because it characterizes the variance-normalized distance between the centers of the two conditional PDFs of the global test static. However, the DC-based methods provide sub-optimal solutions leading to some performance degradation. In this letter, we propose to use GA to optimize the performance of SDF-based linear cooperative spectrum sensing.

\section{Problem formulation}

The system model of linear SDF-based cooperative spectrum sensing is shown in Fig. 1 where $M$ SUs in the network send their statistical measurement data to a common fusion centre (FC). Each SU in the network serves as a relay as it receives a distinct version of probable $\mathrm{PU}$ transmission and then forwards it to FC. FC receives the SUs signals and performs linear weighted SDF to combine the individual measurements and concludes a final decision on the availability of PU. The sensing task at any arbitrary SU is formulated as the binary hypothesis test,

$$
\begin{aligned}
& H_{0}: X_{i}[n]=W_{i}[n] \\
& H_{1}: X_{i}[n]=g_{i} S[n]+W_{i}[n]
\end{aligned}
$$


where $X_{i}[n]$ is the received sampled signal at the $i^{\text {th }} \mathrm{SU}$ receiver and $i=$ $1,2, \ldots, M, n=1,2, \ldots, K ; K$ is the number of samples of the received signal, $g_{i}$ is the sensing channel gain between the PU and the $i^{\text {th }} \mathrm{SU}, S[n]$ is the PU signal which is assumed to be independent and identically distributed (i.i.d.) Gaussian random process with zero mean and variance, i.e., $S[n] \sim \mathcal{N}\left(0, \sigma_{S}^{2}\right)$, and $W_{i}[n]$ is the $i^{\text {th }}$ sensing channel noise which is assumed to be additive white Gaussian with zero mean and variance $\sigma_{W i}^{2}$, i.e., $W_{i}[n] \sim \mathcal{N}\left(0, \sigma_{W i}^{2}\right)$. All these variances are collected into the vector $\vec{\sigma}_{W}=\left[\sigma_{W 1}^{2}, \sigma_{W 2}^{2}, \ldots, \sigma_{W M}^{2}\right]^{T}$, where $T$ is the transpose of the vector and the sampled signals received at the $M$ SUs are collected into the vector $\vec{X}=\left[X_{1}, X_{2}, \ldots, X_{M}\right]^{T}$. The channel gains of the PU-SU and SU-FC links, $g_{i}$ and $h_{i}$, respectively, are assumed to be constant over each sensing period [6]. The reporting channel noises $\left\{N_{i}\right\}$ are assumed to be zero mean and spatially uncorrelated additive white Gaussian with variances $\left\{\delta_{i}^{2}\right\}$ which are collected into the vector $\vec{\delta}=$ $\left[\delta_{1}^{2}, \delta_{2}^{2}, \ldots, \delta_{m}^{2}\right]^{T}$. The energy contents $\left\{Z_{i}\right\}$ of signals of the $M$ SUs, $\left\{Y_{i}\right\}$, received by $\mathrm{FC}\left\{Y_{i}\right\}$ are then estimated as $Z_{i}=\sum_{n=1}^{K}\left|Y_{i}[n]\right|^{2}$. Next, all the individual test statistics $\left\{Z_{i}\right\}$ are used by FC to formulate a global test statistic, $Z$, which can be expressed as

$$
Z=\sum_{i=1}^{M} \omega_{i} Z_{i}=\vec{\omega}^{T} \vec{Z}
$$

where $\vec{Z}=\left[Z_{1}, Z_{2}, \ldots, Z_{M}\right]^{T}$ and $\vec{\omega}=\left[\omega_{1}, \omega_{2}, \ldots, \omega_{M}\right]^{T} ; \omega_{i} \geq 0$ is the weighting coefficients vector assigned by FC. Under this framework, the probability of detection for a given probability of false alarm $\bar{P}_{f}$ can be expressed as [6]

$$
P_{d}=Q\left(\frac{Q^{-1}\left(\bar{P}_{f}\right) \sqrt{\vec{\omega}^{T} \sum_{H_{0}} \vec{\omega}}-\vec{\omega}^{T} \vec{\theta}}{\sqrt{\vec{\omega}^{T} \sum_{H_{1}} \vec{\omega}}}\right)
$$

Where $Q(x)=\int_{x}^{+\infty} \frac{1}{\sqrt{2 \pi}} e^{-t^{2} / 2} d t, \vec{\theta}=\left[\theta_{1}, \theta_{2}, \ldots, \theta_{M}\right]^{T} ; \theta_{i}=K P_{R i}\left|g_{i}\right|^{2}\left|h_{i}\right|^{2} \sigma_{S}^{2}$; $P_{R i}$ is the transmit power of the $i^{\text {th }}$ relay, $\sum_{H_{0}}$ and $\sum_{H_{1}}$ are covariance matrices defined as $\sum_{H_{0}}=\operatorname{diag}\left(2 K \sigma_{0, i}^{4}\right)$ and $\sum_{H_{1}}=\operatorname{diag}\left(2 K\left(P_{R i}\left|g_{i}\right|^{2}\left|h_{i}\right|^{2} \sigma_{S}^{2}+\sigma_{0, i}^{2}\right)^{2}\right)$, respectively; $\sigma_{0, i}^{2}=P_{R i}\left|h_{i}\right|^{2} \sigma_{W i}^{2}+\delta_{i}^{2}$ that is the variance of the $Y_{i}$ under $H_{0}$, and $\operatorname{diag}($.$) is a square diagonal matrix with the elements of a given vector$ on the diagonal. In this letter, equation (3) forms the detection performance criterion of the linear SDF-based sensing scheme. It is clear that $P_{d}$ is very much dependent on $\vec{\omega}$. Thus, the optimal weighting vector is defined as the one that maximizes $P_{d}$. However, if $\vec{\omega}^{*}$ is an optimal solution which maximizes $P_{d}$, there will be an unlimited number of optimal solutions of the form $\psi \vec{\omega}^{*}$, where $\psi$ is any real scaling factor. Therefore, additional constraint is proposed to limit the number of optimal solutions and reduce the search space on which the GA works. The optimal weighting vector of the constraint optimization problem becomes

$$
\begin{gathered}
\vec{\omega}^{*}=\underset{\omega}{\arg \max } P_{d}(\vec{\omega}) \\
\text { s.t. }\|\vec{\omega}\|_{2}=1
\end{gathered}
$$

where $\|.\|_{2}$ in the constraint is 2-norm of the vector $\vec{\omega}$. 


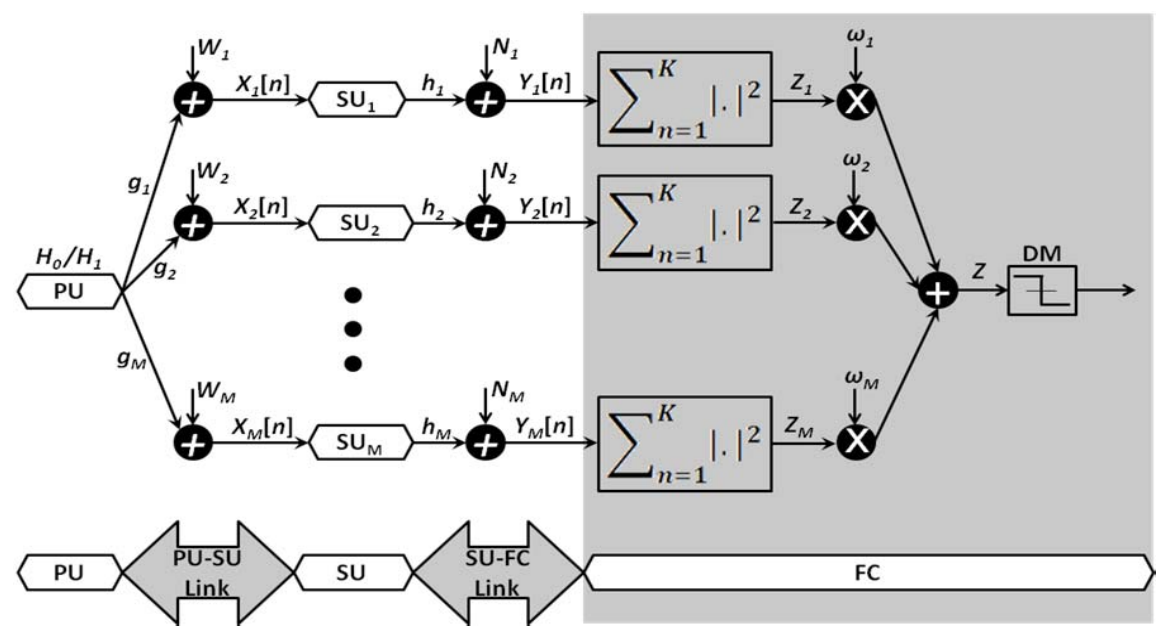

Fig. 1. CR system model with linear SDF-based cooperative spectrum sensing.

\section{Proposed genetic algorithm-based weighting scheme}

GAs are well-known as powerful and broadly applicable stochastic search and optimization techniques [7]. In binary GA, solutions are represented by binary strings (chromosomes) evolve in a manner similar to the genetic growth. A population of strings $P(t)$ at iteration (generation) $t$ is randomly initialized giving a diverse range of possible solutions (candidates). Each binary string consists of one or more variables (traits) encoded by equal or possibly unequal number of bits (genes). The binary-coding search space (genotype) is then accurately decoded to a solution space (phenotype) based on one-toone mapping to ensure no trivial solutions. Each of these possible solutions is evaluated and given a fitness score. The selection operator is then invoked to select the more fit chromosomes (individuals) from the parent population. This is, in Darwinian terms, performing a 'survival of the fittest' operation on the population. The selected population then forms the basis of a mating pool and undergoes stochastic transformations by means of genetic operations to form new individuals. The genetic operators are: crossover, which creates new offspring strings by combining parts (alleles) from two parent individuals, and mutation, which creates new individuals by randomly making changes in the genes of a single individual to encourage diversity. The population of the new individuals (children) resulting from crossover and mutation are called offspring population $C(t)$. It is worth to notice that genetic operators (crossover and mutation) perform essentially a blind search whereas selection operators provide the driving force to hopefully steer the genetic search toward the desirable region of the solution space. Once $C(t)$ is formed, a new population is formed, by combining $C(t)$ with the fittest chromosomes of the parent population, and re-evaluated. The evolutionary process continues and after several predefined number of generations, the algorithm converges to the best individual which hopefully represents an optimal solution to the problem at hand. In this letter, the possible settings of the weighting coefficients vector of the linear SDF scheme are represented by 
chromosomes of $M$ variables each, and each variable is encoded by certain number of bits. The initial population of chromosomes is generated randomly and each chromosome is decoded and normalized to satisfy the constraint in (4). As our objective is to maximize $P_{d}$, we utilize it as the fitness function (i.e. $\left.f(\omega)=P_{d}(\omega)\right)$ used to evaluate the quality of the chromosomes. The proposed GA-assisted SDF-based optimization algorithm is depicted in Table I.

Table I. GA-assisted SDF-based algorithm.

Step 1: Set $t=0$ and randomly generate a population $P(t)$ of pops
chromosomes each of which is of $\left(M^{*} n b i t s\right)$ bits long, where $M$ is the
number of weighting coefficients (i.e. number of SUs in the network) and
nbits is the number of bits to represent each coefficient.
Step 2: Decode each chromosome in the random population into its
corresponding weighting coefficients vector $\vec{\omega}_{j}=\left[\omega_{j 1}, \omega_{j 2}, \ldots, \omega_{j M}\right]^{T}$,
where $\omega_{j i} \in[0,1], i=1,2, \ldots, M$ and $j=1,2, \ldots$, pops.

Step 3: Normalize the weighting coefficients vector through dividing $\vec{\omega}_{j}$ by its 2-norm such that $\vec{\omega}_{j}^{*}=\vec{\omega}_{j} /\left(\sum_{i=1}^{M}\left(\omega_{j i}\right)^{2}\right)^{1 / 2}$ so that the constraint $\left\|\vec{\omega}_{j}^{*}\right\|_{2}=1$ is satisfied.

\begin{tabular}{l} 
Step 4: Compute the fitness score of every normalized decoded weighting \\
vector, $\vec{\omega}_{j}^{*}$, and rank their corresponding chromosomes based on their \\
fitness scores and identify the best $\lfloor$ pops $*$ elite $\rfloor$ chromosomes, where elite \\
is a parameter determines a fraction of pops, i.e. elite $\in[0,1)$, and $\lfloor\rfloor$. \\
denotes floor operation. \\
Step 5: Update $t=t+1$ and reproduce $\lceil$ pops $*(1-$ elite $)\rceil$ new chromosomes \\
(candidate solutions) through genetic algorithm operations; selection, \\
crossover, and mutation, where $\lceil$.$\rceil denotes ceiling operation.$ \\
Step 6: Construct new population $P(t)$ by concatenating the newly \\
$\left\lceil p o p s^{*}(1-\right.$ elite $\left.)\right\rceil$ reproduced chromosomes with the best $\lfloor$ pops $*$ elite $\rfloor$ \\
found in $P(t-1)$. \\
Step 7: decode and normalize the chromosomes of the new population $P(t)$ \\
as in Step 2 and Step 3, respectively. \\
Step 8: Evaluate the fitness score of each candidate solution as in Step 4. \\
\hline Step 9: if $t$ is equal to a predefined number of generations (iterations), ngene, \\
terminate the algorithm; otherwise go to Step 5.
\end{tabular}

\section{Numerical results}

In this Section, the convergence and detection performance of the proposed GA-assisted scheme are both evaluated. In order to choose an appropriate form of the algorithm to meet the nature of the given problem and to achieve more efficient evolutionary processes, the effect of various GA parameter settings has been analyzed using a set-and-test approach. The GA parameters and operators used in this simulation are set as follows: pops $=30, P_{c}=0.95$, and $P_{m}=0.01$ as in [8] for online applications; where $P_{c}$ is two-point crossover rate and $P_{m}$ is bit-flip mutation rate, nbits $=6$, elite $=0.1$, ngene $=100$, and roulette wheel selection was used. The CR network simulation parameters are $M=20, K=150, \bar{P}_{f}=0.25, P_{R i}=12 \mathrm{dBm}$, and $\left\{g_{i}\right\},\left\{h_{i}\right\}$ are 
randomly-generated so that low SNR environment at SU and FC receivers is realized (SNR $<-10 \mathrm{~dB}$ ). Notice that $\left\{g_{i}\right\},\left\{h_{i}\right\}$ are assumed to be constant over every sensing time period; this is justified by the slow-fading nature over the PU-SU and SU-FC links where the delay requirement is short compared to the channel coherence time in the so-called quasi-static scenario. For the same channel condition, the maximum $P_{d}$ fitness of the best chromosome and the mean fitness of all chromosomes in every generation are both shown in Fig. 2 (a). It can be seen that the proposed GA-assisted scheme converges within the first 50 generations, which is so fast. This means that the computation complexity of the proposed scheme can meet the real-time requirements. The improvement achieved by the evolutionary processes of GA after 44 generations is about $12.75 \%$ which is a considerable gain. The same optimal solution (optimal weighting vector) obtained from the GA operations is used to evaluate the performance of the GA-assisted scheme indexed in Fig. 2 (b) by plotting the receiver operating characteristics (ROC)

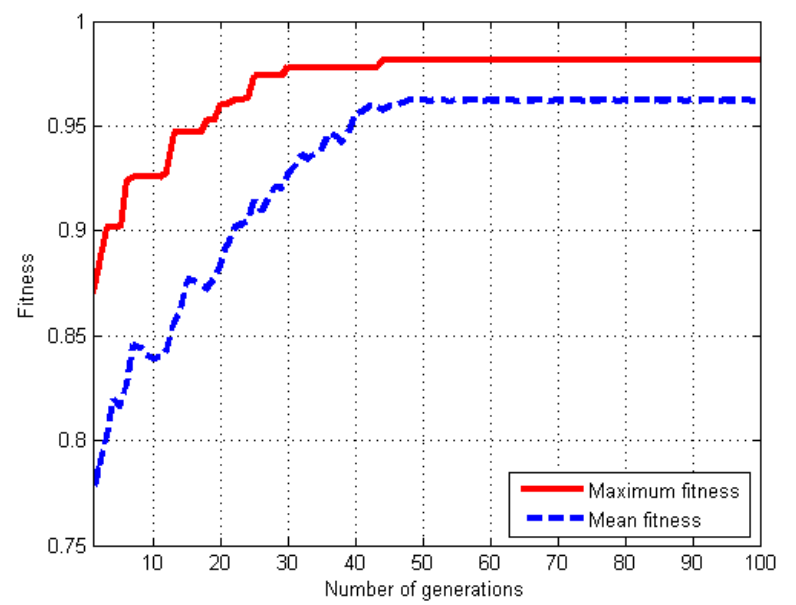

(a)

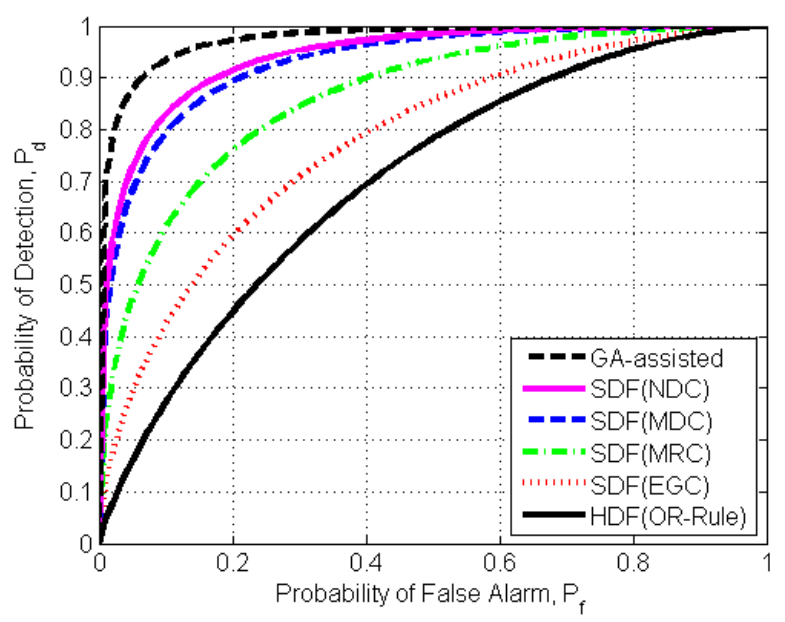

(b)

Fig. 2. Evaluation of convergence performance, (a), and detection performance, (b), of the proposed GAassisted SDF-based scheme. 
curve. Fig. 2 (b) shows a comprehensive detection performance comparison of the GA-assisted scheme and the conventional SDF-based schemes as well as the OR-logic HDF scheme. In the best two shown SDF-based schemes, the weighting coefficients vector is optimized by maximizing the natural deflection coefficient (NDC) and modified deflection coefficient (MDC) [4]. The maximal-ratio combining- (MRC-) based scheme assigns fractional coefficient weights relative to the corresponding SNR values at the SUs' receivers [6]. In Equal gain combination (EGC) scheme, the individual weights assigned to the $M$ SU signals at the FC are all equal [6]. The OR-logic HDF scheme uses different strategy from the one described in Fig. 1 where the SUs in the network process their measurements locally and send their 1-bit decisions to the common FC [3]. Thus, the weighting process used in SDF is not applicable in HDF. The proposed GA-assisted SDF-based scheme uses different methodology where the optimal weighting coefficients vector is obtained by maximizing the probability of detection for a given false alarm probability as in (4). In Fig. 2 (b), it can be seen that our proposed scheme outperforms all other conventional SDF and HDF schemes, which validates the effectiveness of our proposed GA-assisted SDF-based linear cooperative spectrum sensing scheme. Higher probability of detection induces more protection for PUs which is a concrete essential in CR networks.

\section{Conclusion}

In this letter, a GA-assisted SDF-based scheme is used to optimize the weighting coefficients vector used in linear cooperative spectrum sensing. The simulation results show that the proposed scheme has a good convergence speed towards the optimal solutions. The detection performance of the proposed scheme is evaluated and compared with NDC-, MDC-, MRC-, EGC-based SDF schemes as well as the OR-logic HDF scheme. The comparison shows that the proposed scheme can achieve higher probability of detection than all other schemes given the same probability of false alarm.

\section{Acknowledgments}

The authors are very grateful to the Government for funding this work under PKT3/2009 grant. 